\title{
BOLOS EPIDURALES INTERMITENTES PROGRAMADOS PARA MANTENIMIENTO DE LA ANALGESIA DEL TRABAJO DE PARTO: ESTUDIO OBSERVACIONAL, ANALÍTICO DE TIPO COHORTE
}

\author{
Marjorie Lisseth Calderón Lozano${ }^{1}$, Renato Moreno Gonzales², Dante Segura Pinedo ${ }^{3}$, Gunther Vásquez Rojas ${ }^{4}$, \\ Anibal Arenas Velásquez ${ }^{5}$
}

RESUMEN

Objetivo: Evaluar la asociación entre analgesia epidural mantenida mediante Bolos epidurales intermitentes programados (BEIP) y las complicaciones materno-perinatales. Materiales y métodos: Estudio de tipo Cohorte en gestantes entre 18 y 35 años, en trabajo de parto con dilatación cervical de 4 centímetros o más, agrupadas en dos cohortes según administración o no de analgesia epidural: En las gestantes del Grupo A, se administró analgesia epidural en bolo, con mantenimiento mediante BEIP con bupivacaína $0.0625 \%$ y fentanilo 25 ug en 10cc cada 60 minutos; en las gestantes del grupo B, no se administró analgesia epidural. Resultados: La duración del periodo de dilatación y expulsivo fue mayor en el grupo que recibió analgesia epidural de (532.91 \pm 254 minutos) y de (429.19 \pm 311 minutos) en el que no recibió; el tiempo del periodo expulsivo fue (16.97 \pm 16 minutos) y (11.76 \pm 10 minutos) en el grupo $B,(p<0.05)$; menor incidencia de desgarros perineales de primer grado en el grupo que recibió analgesia ( $16.12 \%$ VS $25.35 \% ; p=0.014)$. No se encontraron diferencias en la vía de culminación del parto, puntuación de APGAR, necesidad de reanimación neonatal e ingreso a Unidad de cuidados intensivos neonatales (UCIN). Conclusiones: Las gestantes que recibieron analgesia epidural mediante BEIP tuvieron una mayor duración del periodo de dilatación y del expulsivo, menor incidencia de desgarros perineales, sin incrementar la morbilidad neonatal.

Palabras Claves: analgesia epidural; trabajo de parto; bolos epidurales intermitentes programados; bolos epidurales automatizados (Fuente: DeCS BIREME)

\section{PROGRAMMED INTERMITTENT EPIDURAL BOLUS TECHNIQUE FOR LABOUR ANALGESIA: COHORT STUDY}

\begin{abstract}
Objective: Neuroaxial analgesia is the most effective technique to treat labor pain, however, there are still concerns regarding the undesirable effects. Objective: To evaluate the association between epidural analgesia maintained by programmed intermittent epidural boluses (PEIB) and maternal perinatal complications. Materials and methods: Observational, analytical study of Cohort type in pregnant women between 18 and 35 years, in labor with cervical dilation of 4 centimeters or more, grouped in two cohorts according to administration or not of epidural analgesia: In pregnant women of Group A, administered bolus epidural analgesia, with maintenance by BEIP with bupivacaine $0.0625 \%$ and fentanyl 25 ug in 10cc every 60 minutes; in pregnant women of group B, epidural analgesia was not administered. Results: The duration of the dilation and expulsion period was longer in the group that received epidural analgesia of (532.91 \pm 254 minutes) and (429.19 \pm 311 minutes) in which they did not receive; the time of the expulsion period was (16.97 \pm 16 minutes) and (11.76 \pm 10 minutes) in group B, $(p<0.05)$; lower incidence of first degree perineal tears in the group that received analgesia $(16.12 \%$ VS $25.35 \% ; p=0.014)$. No differences were found in the route of completion of labor, APGAR score, need for neonatal resuscitation and admission to Neonatal intensive care unit (NICU). Conclusions: Pregnant women who received epidural analgesia through BEIP had a longer duration of the period of dilation and expulsion, less incidence of perineal tears, without increasing neonatal morbidity.
\end{abstract}

Keywords: Epidural analgesia; labor; programmed intermittent epidural boluses; automated epidural boluses. (Source: MeSH NLM).

\section{INTRODUCCIÓN}

El dolor de parto es una de las experiencias más duras y traumáticas que afrontan las mujeres durante su vida, ${ }^{1}$ su impacto perjudicial en el bienestar de las madres ha dado lugar al desarrollo de diferentes políticas de salud. ${ }^{2}$

La analgesia neuroaxial es la técnica más efectiva para tratar el dolor de parto, sin embargo, a pesar del uso creciente y las ventajas innegables, hay preocupaciones con respecto a los efectos indeseables sobre la progresión del trabajo de parto y los resultados neonatales. Es así que los regímenes de mantenimiento también han evolucionado desde bolos manuales hasta infusiones continuas, analgesia epidural controlada por la paciente (PCEA) con o sin infusión de fondo. Una alternativa son los bolos epidurales intermitentes programados: método automatizado de administración de anestésico

\footnotetext{
1-5 Médico Asistente del Departamento de Anestesiología, Analgesia y Reanimación del Instituto Nacional Materno Perinatal. Lima-Perú. Citar como: Calderón ML, Moreno R, Segura D, Vásquez G, Arenas A. Bolos epidurales intermitentes programados para mantenimiento de la analgesia del trabajo de parto: estudio observacional, analítico de tipo cohorte. Rev Peru Investig Matern Perinat 2020; 9(3):28-34 DOI https://doi.org/10.33421/inmp.2020194
}

Recibido: 11-08-20 Aprobado: 30-09-20 
local epidural con o sin opioides, la técnica proporciona bolos fijos a intervalos programados. ${ }^{3}$ Un meta análisis de estos estudios comparativos encontró una reducción estadísticamente significativa en el uso de anestésicos locales y mayor satisfacción materna con BEIP, pero también existe la posibilidad de que este método disminuya la tasa de parto instrumentado y la necesidad de intervenciones adicionales. ${ }^{4}$

El objetivo del presente estudio fue evaluar la asociación entre analgesia epidural mantenida por BEIP durante el trabajo de parto y las complicaciones materno perinatales según monitoreo electrónico fetal alterado, prolongación del trabajo de parto, desgarro vaginal, terminación del parto en cesárea, depresión al nacer, reanimación neonatal e ingreso a Unidad de cuidados intensivos neonatales (UCIN), en gestantes en trabajo de parto. Los datos obtenidos pueden ayudar a los médicos a corregir los conceptos erróneos con respecto a los efectos adversos de la analgesia neuroaxial y fomentar las nuevas estrategias de mantenimiento para un mejor resultado del parto.

\section{MATERIALES Y MÉTODOS}

Es un estudio observacional, analítico de tipo Cohorte, aprobado por los comités de ética y metodología del Instituto Nacional Materno Perinatal (INMP) de Lima Perú con el número de oficio 0119-2017-DG-N 044-OEAIDE/ INMP, el 25 de mayo del 2017 y ejecutado en gestantes con trabajo de parto que fueron atendidas en el servicio de Centro obstétrico del INMP durante junio del 2017 a junio del 2018.

Se seleccionó gestantes entre 18 y 35 años, multíparas o primíparas que tuvieron trabajo de parto con dilatación cervical de 4 centímetros o más, con dinámica uterina útil y cuello favorable al tacto vaginal, que presentaron monitoreo electrónico fetal patrón 1, con administración de analgesia epidural durante el trabajo de parto, y aceptaron participar del estudio previo consentimiento informado. Se excluyeron a las gestantes con antecedentes obstétricos desfavorables, signos de estado fetal no satisfactorio, inestabilidad hemodinámica, hemoglobina menor a $10 \mathrm{~g} / \mathrm{dl}$ y plaquetas menores de $150000 / \mathrm{mm}^{3}$.

Ingresaron al estudio todas las participantes que cumplieron los criterios de selección. Posteriormente fueron agrupadas en dos cohortes según administración o no de analgesia epidural: Las gestantes del grupo A donde fue indicada la analgesia durante el trabajo de parto y las gestantes del grupo B donde no fue indicada analgesia alguna.

Ambos grupos fueron monitorizados con oximetría de pulso, presión arterial no invasiva y monitorización cardiotocográfica externa. En las gestantes del Grupo A, se registró el cumplimiento de la técnica de administración de analgesia, así como la dosis prescrita vía catéter epidural, en la cual el bolo de inicio fue bupivacaína al $0,125 \%$ más fentanilo 50 ug en un volumen total de 20cc y dosis de mantenimiento 60 minutos después del bolo inicial mediante BEIP con bupivacaína $0.0625 \%$ y fentanilo 25 ug en $10 \mathrm{cc}$ cada hora hasta el parto. Se valoró el dolor mediante la escala numérica verbal (ENV), en una escala de 0-10 (0 representa la ausencia de dolor y 10 el dolor más intenso), considerándose los rescates con bupivacaína $0,125 \%$ en volumen de $5 \mathrm{cc}$, cuando la paciente presentaba ENV mayor a 3. Se usó la bomba Infusomat Space de Braun. Posteriormente a la administración o no de la analgesia, se realizó un monitoreo electrónico fetal y de signos vitales maternos durante todo el trabajo de parto.

Se evaluaron los siguientes resultados, la media de la duración del trabajo de parto en minutos, la presencia o ausencia de desgarros perineales, la proporción de la terminación del parto en cesárea, depresión al nacer mediante puntuación de APGAR al primer minuto y a los cinco minutos, necesidad o no de reanimación neonatal e ingreso a UCIN.

El tamaño de la muestra se determinó, considerando un riesgo alfa de 0.05 y un riesgo beta de 0.2 en un contraste bilateral, una razón entre el número de no expuestos y de expuestos de 1/1. Por lo cual, se precisó 300 participantes en el grupo de expuestos a la analgesia epidural y 300 en el de no expuestos.

El procesamiento de los datos inició con el análisis exploratorio descriptivo univariado con medidas de resumen de posición central y de variabilidad para las variables de naturaleza cuantitativa, mientras que para las variables cualitativas se utilizó las distribuciones de frecuencias y porcentuales, dichos estadísticos calculados estuvieron segmentados según la administración de la analgesia epidural.

Para el análisis bivariado cuantitativo se utilizó pruebas paramétricas de comparación de medias (t student), teniendo en consideración el coeficiente de variación menor al $30 \%$, para los casos superiores a dicho valor se utilizó pruebas no paramétricas para la comparación de mediana (test U- Mann Whitney) dado que dicho indicador no se ve afectado por la presencia de datos extremos; y para el caso del análisis bivariado cualitativo se utilizó pruebas de comparación proporcional mediante Chi cuadrado. Todas las pruebas inferenciales fueron bilaterales y tuvieron un nivel de confianza del $95 \%$. El procesamiento fue realizado en el software estadístico SPSS versión 25, STATA versión 15 y la hoja de cálculo Excel.

\section{RESULTADOS}

Un total de 600 pacientes fueron consideradas potencialmente elegibles para el estudio; dos de ellas desistieron de enrolarse en éste, siendo 598 las 
participantes confirmadas e incluidas. De éste grupo fueron excluidas 24 gestantes. Se conformaron dos grupos con 574 participantes: grupo A con 274 participantes que recibieron analgesia durante el trabajo de parto y grupo $B$ con 300 participantes que no recibieron analgesia durante el trabajo de parto. (Fig. 1).

Las características demográficas fueron similares entre los grupos. Las características obstétricas difirieron en la paridad, siendo mayor el grupo de primíparas en las gestantes que recibían analgesia durante el trabajo de parto, y predominando las multíparas en el grupo que no recibieron analgesia durante el trabajo de parto (Tabla 1).

Estas pacientes tuvieron un seguimiento desde el ingreso a centro obstétrico hasta el alta hospitalaria, que en la mayoría de las pacientes fue 24 a 36 horas después del parto. En relación a lo resultados maternos (Tabla 2), se encontró diferencia estadísticamente significativa en la duración del trabajo de parto, el
Tabla 1. Características demográficas, clínicas y osbtétricas

\begin{tabular}{llc}
\hline \multicolumn{1}{c}{ CARACTERÍSTICAS } & \multicolumn{1}{c}{$\begin{array}{c}\text { GRUPO A } \\
(\mathbf{n = 2 7 4 )}\end{array}$} & $\begin{array}{c}\text { GRUPO B } \\
(\mathbf{n = 3 0 0 )}\end{array}$ \\
\hline Edad maternal (años) & $24.18 \pm 4.84$ & $25.54 \pm 4.80$ \\
Índice de masa corporal & $28.35 \pm 3.70$ & $28.82 \pm 3.39$ \\
Hemoglobina (g/dl) & $12.08 \pm 1.03$ & $12.05 \pm 1.08$ \\
Plaquetas (/mm3) & $258190 \pm 128725$ & $247273 \pm 65762$ \\
Paridad & & \\
Primípara & $63.14 \%$ & $35.22 \%$ \\
Multípara & $36.86 \%$ & $64.78 \%$ \\
\hline
\end{tabular}

Grupo A: se administró analgesia epidural del parto.

Grupo B: no se administró analgesia epidural.

periodo de dilatación y expulsivo fue mayor en el grupo que recibió analgesia epidural (532.91 \pm 254 minutos) y (429.19 \pm 311 minutos) en el grupo sin analgesia; el tiempo del periodo expulsivo fue (16.97 \pm 16 minutos) y (11.76 \pm 10 minutos) en el grupo sin analgesia, $(p<$ 0.05); (Figura 2).

Grupo A: se administró analgesia epidural del parto. Grupo B: no se administró analgesia epidural

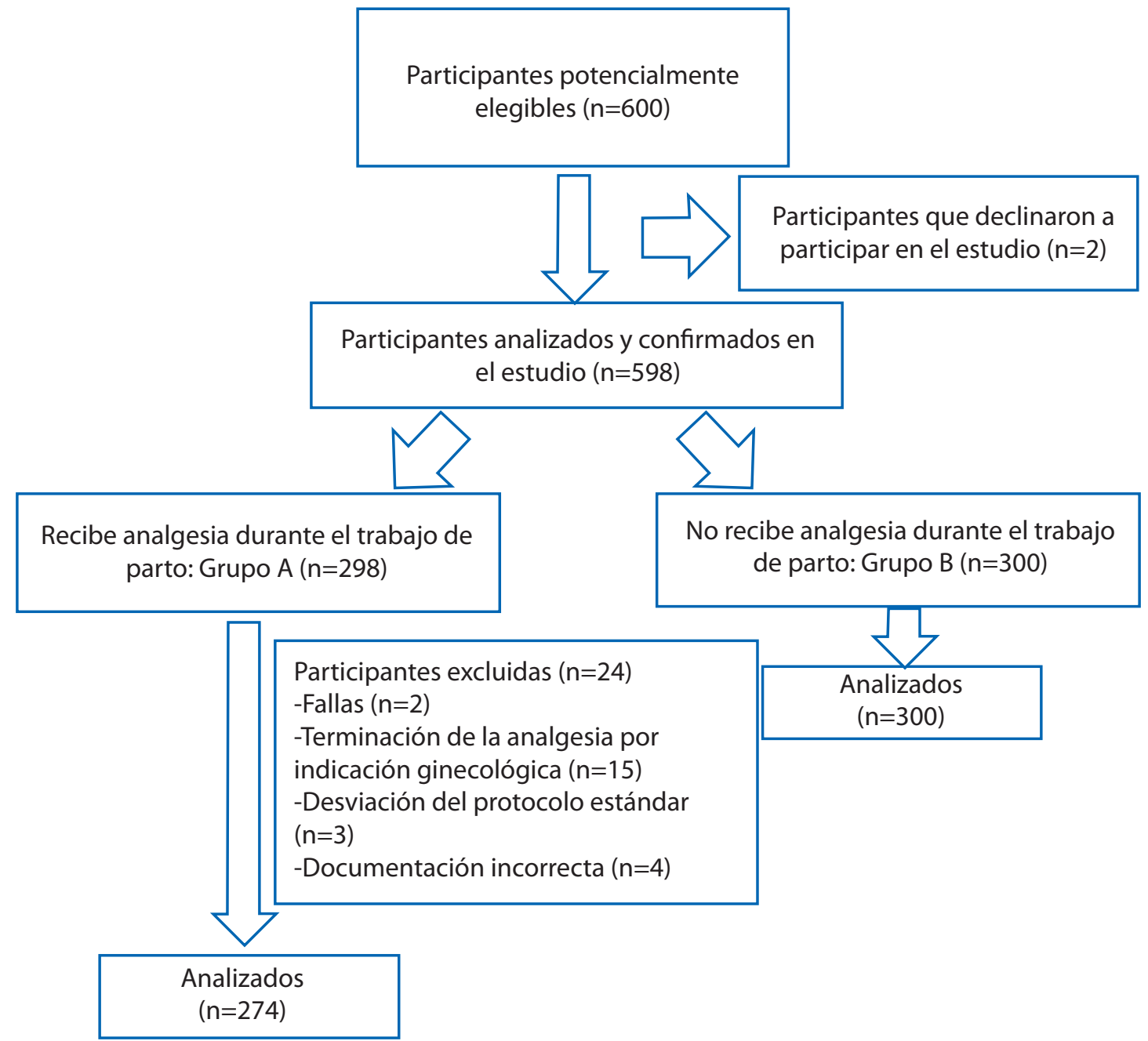

Figura 1. Diagrama de flujo del proceso de selección de las participantes incluidas en el análisis del estudio. 
Tabla 2. Resultados materno-perinatales

\begin{tabular}{|c|c|c|c|}
\hline$(n=274)$ & $\begin{array}{l}\text { Grupo A } \\
(n=300)\end{array}$ & Grupo B & $\begin{array}{l}\text { Valor } \\
\text { de } p\end{array}$ \\
\hline \multicolumn{4}{|c|}{ RESULTADOS MATERNOS } \\
\hline \multicolumn{4}{|c|}{ Fases del Trabajo de Parto } \\
\hline $\begin{array}{l}\text { Tiempo de dilatación } \\
\text { (min) }\end{array}$ & $532.91 \pm 254.05$ & $429.19 \pm 311.30$ & 0.000 \\
\hline $\begin{array}{l}\text { Tiempo de expulsivo } \\
\text { (min) }\end{array}$ & $16.97 \pm 16.08$ & $11.76 \pm 10.03$ & 0.000 \\
\hline Nulíparas & 18.41 & 14.70 & 0.005 \\
\hline Multíparas & 15.65 & 11.17 & 0.007 \\
\hline \multicolumn{4}{|c|}{ Desgarros Perianeales } \\
\hline No & $79.12 \%$ & $70.07 \%$ & 0.043 \\
\hline I Grado & $16.12 \%$ & $25.35 \%$ & 0.014 \\
\hline II Grado & $4.40 \%$ & $3.52 \%$ & 0.535 \\
\hline III Grado & $0.37 \%$ & $0.35 \%$ & 0.956 \\
\hline IV Grado & $0 \%$ & $0 \%$ & \\
\hline Término de parto & & & 0.148 \\
\hline Vaginal (\%) & $91.24 \%$ & $94.35 \%$ & \\
\hline Cesárea (\%) & $8.76 \%$ & $5.65 \%$ & \\
\hline
\end{tabular}

\section{RESULTADOS NEONATALES}

\begin{tabular}{|c|c|c|c|}
\hline \multicolumn{4}{|c|}{ Apgar al minuto } \\
\hline$\leq 3$ & $0.73 \%$ & $0.33 \%$ & 0.507 \\
\hline 4 a 6 & $2.93 \%$ & $3.32 \%$ & 0.788 \\
\hline$\geq 7$ & $96.34 \%$ & $96.35 \%$ & 0.996 \\
\hline \multicolumn{4}{|c|}{ Apgar a los 5 minutos } \\
\hline$\leq 3$ & $0 \%$ & $0 \%$ & \\
\hline 4 a 6 & $0 \%$ & $0 \%$ & \\
\hline$\geq 7$ & $100 \%$ & $100 \%$ & \\
\hline \multicolumn{4}{|c|}{ Reanimación neonatal } \\
\hline No & $98.16 \%$ & $99.00 \%$ & 0.391 \\
\hline Sí & $1.84 \%$ & $1.00 \%$ & 0.391 \\
\hline \multicolumn{4}{|c|}{ Ingreso a Unidad de Cuidados Intensivos neonatales } \\
\hline No & $99.25 \%$ & $97.34 \%$ & 0.133 \\
\hline Sí & $0.75 \%$ & $2.66 \%$ & 0.133 \\
\hline
\end{tabular}

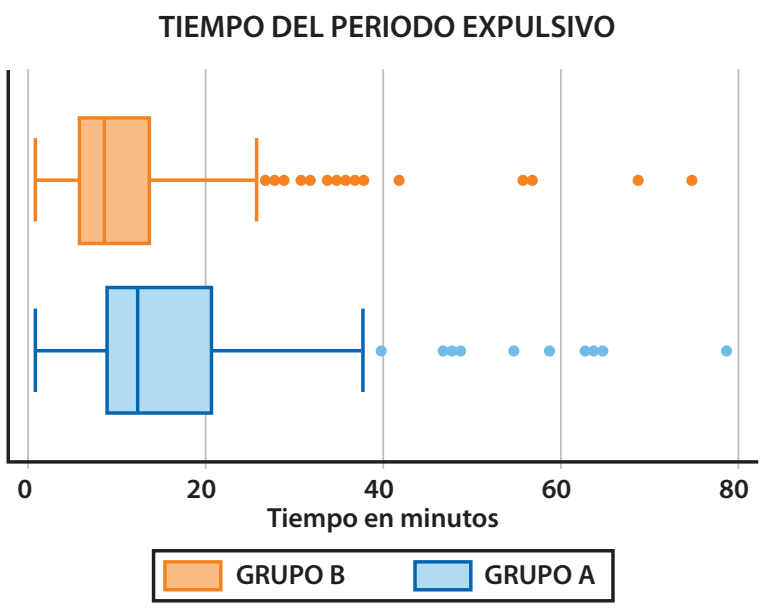

Figura 2. Box Plot de comparación entre ambos grupos de duración en minutos del segundo periodo del trabajo de parto. Grupo A: se administró analgesia epidural del parto. Grupo B: no se administró analgesia epidural

No se encontró diferencia estadística $(p=0.148)$ en la vía de culminación del parto, comparando parto vaginal con parto por cesárea entre ambos grupos (Figura 3). Por otro lado, se encontró menor incidencia de desgarros perineales de primer grado durante el trabajo de parto en el grupo que recibió analgesia (16.12 \% VS 25.35\%; $p=0.014)$ (Figura 4).

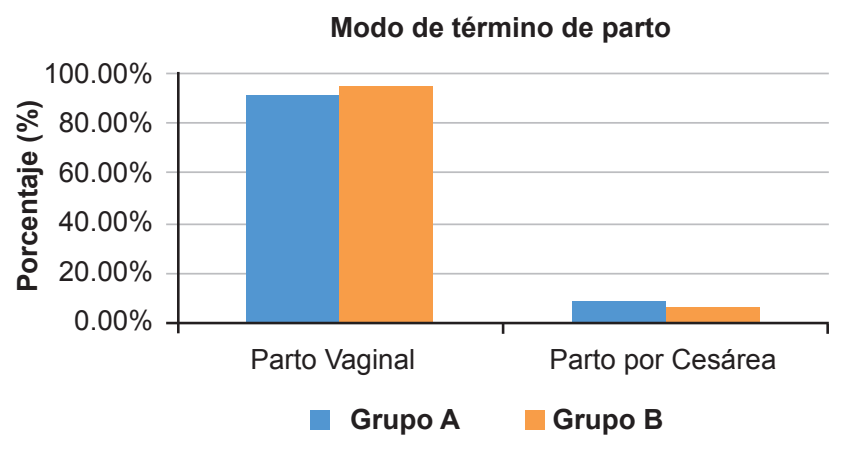

Figura 3: Comparación entre ambos grupos en el modo de término del embarazo (parto vaginal versus parto por cesárea). Grupo A: se administró analgesia epidural del parto. Grupo B: no se administró analgesia epidural.

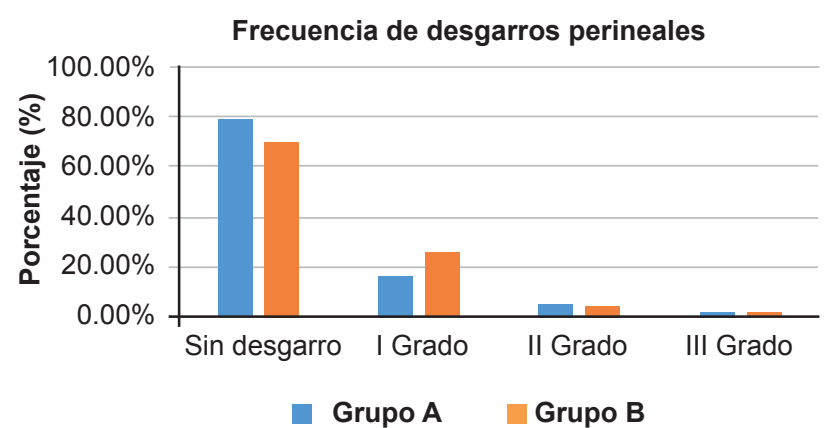

Figura 4: Comparación entre ambos grupos en la frecuencia y tipo de desgarros perineales. Grupo A: se administró analgesia epidural del parto. Grupo B: no se administró analgesia epidural 


\section{Comparación de puntuaciones del Test de APGAR}

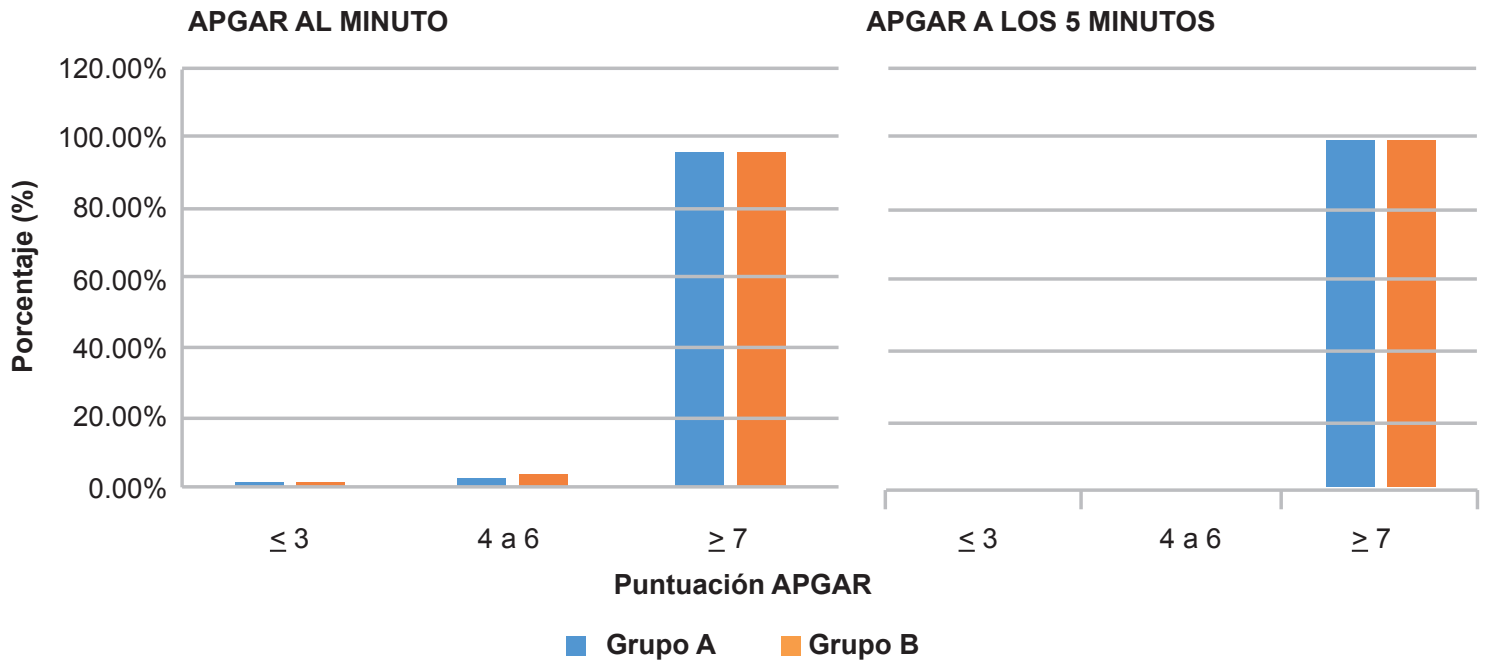

Figura 5: Comparación entre ambos grupos en la puntuación de la escala de APGAR al minuto y a los 5 minutos. Grupo A: se administró analgesia epidural del parto. Grupo B: no se administró analgesia epidural

En relación a los resultados neonatales (Figura 5), en la puntuación de APGAR no se encontraron diferencias estadísticamente significativas al primer y quinto minuto. Tampoco se encontró diferencia estadística en la necesidad de reanimación neonatal e ingreso a $\mathrm{UCl}$ neonatal.

\section{DISCUSIÓN}

En el trabajo de parto, es tan importante proveer analgesia de calidad a las gestantes como evitar la repercusión materna o neonatal negativa. Los actuales regímenes de mantenimiento de la analgesia neuroaxial, con dosis ultra diluidas de anestésicos locales y concentraciones bajas de opioides mediante bolos epidurales intermitentes programados han permitido disminuir los efectos no deseados de la analgesia durante el trabajo de parto. Entre los principales resultados en este estudio, se evidenció diferencias estadísticamente significativas en la duración del primer y segundo estadio del trabajo de parto y el porcentaje de desgarros perineales.

En cuanto a la duración de los periodos del trabajo de parto, Hernández y col., realizaron un estudio de cohortes restrospectivo, en gestantes nulíparas y multíparas que recibían analgesia epidural( $A E)$, a quienes se les administraba ropivacaína a $1 \mathrm{mg} / \mathrm{ml}$ y $2 \mathrm{ug} / \mathrm{ml}$ de fentanilo, mediante una bomba de infusión continua, encontrándose que el tiempo del primer estadio del trabajo de parto fue mayor en las pacientes que recibían analgesia epidural, con una media de 270.5 minutos versus 94.4 minutos en las que no recibían AE $(p<0.001)$; mientras que la duración del segundo estadio de parto fue 78.4 minutos en el grupo que recibió $\mathrm{AE}$ versus 19.3 minutos en el grupo que no recibió $(p<0.001)^{5}$.
En el meta análisis de Cochrane, publicado en el 2018, se comparó la duración del primer estadio del trabajo de parto, que incluyeron 2 estudios con una población de 96 pacientes en el grupo que recibió analgesia epidural y 93 en el grupo que no la recibió o se les administró placebo, no encontrándose diferencias estadísticamente significativas, con un IC $(-186.26,76.09)$. Con respecto al segundo estadio del trabajo de parto, se incluyeron 4 estudios con una población de 173 pacientes en el grupo que recibió analgesia epidural y 171 en el grupo que no recibió o se les administro placebo, tampoco se encontró diferencias estadísticamente significativas, con un IC $(-6.12,21.45){ }^{6}$ Estos resultados contrastan con los resultados obtenidos en nuestro estudio, cuya duración del primer y segundo periodo del trabajo de parto son mayores en las gestantes que recibieron analgesia epidural $(p<0.05)$. Si bien es cierto, la duración del primer y segundo periodo fue mayor en el grupo A en 103.72 minutos y 5.21 minutos respectivamente, estos resultados no aumentaron la tasa de cesárea, ni afectaron los resultados neonatales, como lo veremos a continuación.

También se encontró diferencia estadística en el porcentaje de desgarros perineales tras el parto (20.88\% versus $29.3 \%, p=0.043$ ), a expensas de desgarro de primer grado, es decir lesión que se limita a la piel perineal y/o la mucosa vaginal. Las pacientes que recibieron analgesia de parto tuvieron una menor proporción de estos $(16.12 \%$ versus $25.35 \%, p=0.014$ ). No se encontraron diferencias en la incidencia de desgarros de segundo, tercer y/o cuarto grado.

La incidencia de trauma perineal de algún grado tras el parto varía a nivel mundial del 30 al $75 \%$, en contraste con nuestra muestra en la cual encontramos una proporción menor $(20 \%$ al $29 \%)$ y la incidencia de desgarros 
perineales severos (desgarros de tercer y cuarto grado) van desde el $0.8 \%$ al $3 \% .^{7}$ Este tipo de laceraciones severas generan morbilidad en el puerperio e incluso están asociadas a depresión pos parto. ${ }^{8}$

La analgesia epidural ha sido asociada en algunas publicaciones a aumento de parto instrumentado y mayor proporción de desgarros perineales. Por otro lado, múltiples publicaciones han concluido que la analgesia epidural no predispone a este tipo de injurias y, es más, puede ser un factor protector. Bauman y col. en el 2007, en una cohorte de 40000 gestantes nulíparas en labor de parto encontraron que hubo una reducción en la incidencia de desgarros perineales en las pacientes que recibieron analgesia neuroaxial (OR 0.66, IC al 95\% de $0.57-0.77$ ) y más recientemente Ramm y col. en el 2018 , en una cohorte retrospectiva de 22000 partos vaginales encontraron que la analgesia neuroaxial disminuyó la probabilidad de laceraciones perineales y del esfínter anal (OR de 0.70, IC al 95\% y 0.59-0.82)..$^{8}$ Estos resultados son consistentes con los nuestros tal vez debido a que el factor protector asociado a desgarros perineales de la analgesia epidural se ha asociado al uso de concentraciones bajas de anestésico local para el mantenimiento de la analgesia, como es nuestro caso (bupivacaína al 0,0625\%).

Sobre la relación de la analgesia epidural y la vía del término del parto, ha existido siempre controversias, sin embargo el meta análisis Cochrane publicado en el $2018,{ }^{6}$ reviso 5 estudios con un total de 578 pacientes ( 289 recibieron analgesia epidural y 289 recibieron placebo o no recibieron analgesia); encontrando menor cantidad de pacientes que terminaron el parto por cesárea $(3.8 \%)$ entre las que recibieron analgesia epidural, frente a $8.3 \%$ entre las que no recibieron analgesia, (promedio RR 0.46 , $95 \%$ IC 0.23 al 0.90 ), resultados similares encontramos en nuestro estudio, con un $8.76 \%$ de cesáreas en el Grupo A y un $5.65 \%$ en el grupo B, aunque el porcentaje en el grupo A es mayor, esto no tuvo significancia estadística. $(p=0.148)$. Queda como tarea al futuro abordar las causas de la indicación de cesárea en ambos grupos y su relación o no con la analgesia epidural.

Con respecto a la puntuación de APGAR, Genc y col., en un estudio controlado randomizado de 95 pacientes, en el cual comparó resultados neonatales de 2 grupos (Analgesia Epidural vs No analgesia), no encontró diferencia estadística en la puntuación de Apgar al 1er minuto ( $p=0.613)$, ni a los 5 minutos $(p=0.451)$. Resultados similares fueron encontrados en nuestra investigación ${ }^{9}$.

Herrera-Gómez y col., en el 2015; realizaron un estudio retrospectivo tipo cohortes, en el cual se observó en las usuarias de analgesia, un aumento en las puntuaciones bajas de Apgar, valores más bajos de $\mathrm{pH}$ en partos vaginales normales y mayores necesidades de reanimación neonatal ${ }^{10}$. Aun así, sus resultados en términos de mortalidad y encefalopatía hipóxico-isquémica en los neonatos a estudiar no son unánimes. ${ }^{11,12}$ También se detectó una necesidad más frecuente de reanimación, ya sea básica o avanzada, en neonatos expuestos a analgesia epidural que contrasta con nuestro trabajo que no encontró diferencia estadísticamente significativa $(p=0.391)$ en la necesidad de reanimación neonatal ( $1.84 \%$ en el grupo A y $1.00 \%$ en el grupo B).

Con respecto a el ingreso a UCIN, en el estudio realizado por Deshmukh y $\mathrm{col}^{13}$, no hubo un aumento en la tasa de admisiones en la UCIN tanto en el grupo de estudio como en el de control, coincidiendo con los resultados de nuestro trabajo, al no encontrar diferencia estadística $(p=0.133)$ en el ingreso a UCIN de $0.75 \%$ del grupo con analgesia y de $2.66 \%$ sin analgesia.

En el estudio de Herrera-Gómez y col, en el 2015 no se mostró los datos sobre la causa de ingreso a la UCIN; sin embargo, se encontró un mayor porcentaje de recién nacidos admitidos en el grupo de analgesia epidural que en el grupo que no recibió analgesia. Dado el riesgo de salud para el recién nacido y el aumento de los costos hospitalarios involucrados, parece apropiado resaltar este hallazgo, a pesar de no poder especificar su causa, lo que sugiere que verifiquemos los elementos para completar el modelo de recolección de datos en estudios futuros ${ }^{10}$.

Para resumir, como se había comentado las pacientes que recibieron analgesia epidural del parto con BEIP como mantenimiento tuvieron una mayor duración tanto del periodo de dilatación y del expulsivo, sin repercutir en intervenciones adicionales en la madre como son las cesáreas ni aumentar la morbilidad neonatal con la gran ventaja de controlar de una manera efectiva el dolor del parto y disminuir la incidencia de desgarros perineales de primer grado. Cabe resaltar que una de las mayores fortalezas del estudio es la cantidad de pacientes incluidas la cuál es superior en comparación con publicaciones similares. Por otro lado, una de las más grandes limitaciones del estudio, es la ausencia de randomización entre los grupos, debido a que la indicación de analgesia en nuestra institución es en base al criterio obstétrico. Probablemente esto genere la diferencia en número de pacientes nulíparas y multíparas en ambos grupos.

\section{Reconocimientos}

\section{Contribuciones de los autores:}

- Autor 1 (MCL): Concepción del proyecto original, planificación del estudio, obtención de datos, interpretación de los resultados y redacción inicial y final del manuscrito.

- Autor 2 (RMG). Planificación del estudio, obtención de datos, interpretación de los resultados, análisis de los datos y redacción final del manuscrito.

- Autor 3 (DSP): planificación del estudio, redacción de la metodología, interpretación de los resultados y redacción y aprobación final del manuscrito. 
- Autor 4 (AAV): planificación del estudio, obtención de datos, redacción de la introducción interpretación de los resultados aprobación final del manuscrito.

- Autor 5 (GVR): planificación del estudio, redacción de las conclusiones, interpretación de los resultados y redacción y aprobación final del manuscrito.

\section{Asistencia para el estudio:}

A los doctores Carlos Alejandro Gonzales Medina y al Licenciado en Estadística Augusto Elmer Racchumí Vela, de la Oficina de Investigación del Instituto Nacional Materno Perinatal de Lima-Perú.

\section{Presentaciones}

Un resumen del presente manuscrito se presentó como poster digital en el concurso de posters en el Congreso CLASA de Buenos Aires en septiembre 2019.

\section{Agradecimientos}

A la Jefatura del Departamento De Anestesia Analgesia y Reanimación del Instituto Nacional Materno Perinatal Al Dr. Víctor Chura y al Dr. Wilber Ccopa, anestesiólogos del Hospital Santa Rosa de Lima Perú.

Financiamiento: Autofinanciado.

Conflicto de interés: Los autores declaran no tener algún conflicto de intereses

\section{REFERENCIAS BIBLIOGRÁFICAS}

1. Jung $\mathrm{H}$, Kwak $\mathrm{KH}$. Neuraxial analgesia: a review of its efects on the outcome and duration of labor. Korean J Anesthesiol. 2013,65:379-384. doi: http://10.1213/ ANE.0000000000000546.

2. McCauley M, Actis V, Mrema D, Van den Broek N. "We know it's labour pain, so we don't do anything": healthcare provider's knowledge and attitudes regarding the provision of pain relief during labour and after childbirth. BMC Pregnancy Childbirth. 2018; 18: 444. doi: http://10.1186/s12884-018-2076-7.

3. McKenzie CP, Cobb B, Riley ET, Carvalho B. Programmed intermittent epidural boluses for maintenance of labor analgesia: an impact study International. Journal of Obstetric Anesthesia. 2016,26:32-38. doi: http:// 10.1016/j. ijoa.2015.11.005

4. George RB, Allen TK, Habib AS. Intermittent epidural bolus compared with continuous epidural infusions for labor analgesia: a systematic review and meta-analysis.
Anesth Analg 2013;116:133-44. doi: http://10.1213/ ANE.0b013e3182713b26.

5. Hernández A, Rodríguez JJ, Moreno M, Ureña M, Molina M, Gómez J. Epidural Analgesia and Neonatal Morbidity: A Retrospective Cohort Study. Int. J. Environ. Res. Public Health 2018, 15, 2092;1-10. doi: http://10.3390/ijerph15102092.

6. Anim M, Smyth R, Cyna AM, Cuthbert A. Epidural versus non-epidural or no analgesia for pain management in labour. Cochrane Database of Systematic Reviews. 2018. doi: http://10.1002/14651858.CD000331.pub4.

7. Garcia-Lausin L, Perez-Botella M, Duran X, Rodríguez S, Gutierrez MJ, Escuriet R. Relation between Epidural Analgesia and severe perineal laceration in childbearing women in Catalonia. Midwifery. 2019, 70:76-83. doi: http://10.1016/j.midw.2018.12.007.

8. Peralta F, Bradley J. Severe perineal lacerations after vaginal delivery: are they an anesthesiologist's problem? Curr Opin Anesthesiol 2018, 31:000-000. doi: http:// 10.1097/ ACO.0000000000000599.

9. Genc M, Sahin N, Maral J, Celik E, Kar AA, Usar P et al. Does bupivacaine and fentanyl combination for epidural analgesia shorten the duration of labour? Journal of Obstetrics and Gynaecology, 2014; 1-4. doi: http://10.3109/01443615.2014 .991299 .

10. Herrera A., García O, Ramos J, De Luna E, Ruiz C, Ocaña FM. Retrospective study of the association between epidural analgesia during labour and complications for the newborn. Midwifery. 2015;31(6):613-6. doi: http://10.1016/j. midw.2015.02.013.

11. Törnell, S.; Ekéus, C.; Hultin, M.; Håkansson, S.; Thunberg, J.; Högberg, U. Low Apgar score, neonatal encephalopathy and epidural analgesia during labour: A Swedish registrybased study. Acta Anaesthesiol. Scand. 2015, 59, 486-495. doi: http://10.1111/aas.12477.

12. Laughon SK, Berghella V, Reddy UM, Sundaram R, Lu Z, Hoffman MK. Neonatal and maternal outcomes with prolonged second stage of labour. Obstet. Gynecol. 2014, 124: 57-67. doi: http://10.1097/AOG.0000000000000278.

13. Deshmukh V, Ghosh S, Yelikar K, Gadappa S. Effects of Epidural Labour Analgesia in Mother and Fetus. The Journal of Obstetrics and Gynecology of India. 2018;68(2):111-116. doi: http://10.1007/s13224-017-1063-7.

\section{Correspondencia:}

Marjorie Lisseth Calderón Lozano.

Dirección: Calle Raymundo Carcamo 639 Dpto 102. Urb Santa Catalina -La Victoria

de Porres

Correo: marjorie_163@hotmail.com

Teléfono: $51918 \overline{8} 39858$ 